\title{
Valutazione economica dello studio MIRACL
}

Simona de Portu ${ }^{(1)}$, Sabato Montella ${ }^{(1)}$, Simona Cammarota ${ }^{(1)}$, Lorenzo G. Mantovani ${ }^{(1)}$

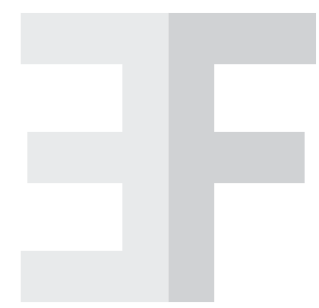

\begin{abstract}
Introduction: the MIRACL study ("Effects of atorvastatin on early recurrent ischemic events in acute coronary syndrome") has evaluated the effects of atorvastatin on early recurrent ischemic events in acute coronary syndromes. It has demonstrated that, for patients with acute coronary syndrome, lipid-lowering therapy with atorvastatin $(80 \mathrm{mg} / \mathrm{die})$ reduces recurrent ischemic events in the first 16 weeks, mostly recurrent symptomatic ischemia requiring rehospitalization.

Aim: the goal of this pharmacoeconomic study is to evaluate the MIRACL study in the Italian context. Methods: the analysis is based on clinical outcome data from the MIRACL study. Clinical outcomes measured in the study include: death, cardiac arrest, nonfatal myocardial infarction (MI), fatal MI, angina pectoris, stroke, congestive heart failure (CHF), and surgical or percutaneous coronary revascularizations. Economic evaluation was carried out conducting a cost/effectiveness analysis, comparing economic and clinical consequences of high doses atorvastatin $(80 \mathrm{mg})$ vs placebo in patients with unstable angina or acute myocardial infarction. We identified and quantified medical costs: drug costs according to the Italian National Therapeutic Formulary and hospitalizations were quantified based on the Italian National Health Service tariffs (2006). Effects were measured in terms of mortality and morbidity reduction (number of deaths, life years gained and frequency of hospitalizations). All direct medical costs were taken from the perspective of the Italian National Health System during a 16-week period. We conducted one and multi-way sensitivity analyses on unit cost and effectiveness. We also conducted a threshold analysis.

Results: the cost of atorvastatin therapy over the 16 weeks period amounted to approximately 162,489 euro per 1,000 patients. The total cost of atorvastatin high dose was about 2.2 millions euro, the incremental cost per patient free from event is 6,601 euro.

Discussion: This evaluation found that atorvastatin therapy is cost-effective. Sensitivity analysis shows that cost consequences parameters are substantially sensitive to fluctuation.
\end{abstract}

Keywords: atorvastatin, acute coronary syndrome, cost, Italian National Health System

\section{INTRODUZIONE}

Lo studio "Effects of atorvastatin on early recurrent ischemic events in acute coronary syndromes" (MIRACL) recentemente ha valutato se il trattamento con atorvastatina $80 \mathrm{mg} / \mathrm{die}$, iniziato $24 / 96$ ore dopo sindrome coronarica acuta, riduca il rischio di morte e gli eventi ischemici non fatali [1].

Lo studio ha dimostrato come il trattamento ipocolesterolemizzante aggressivo con atorvastatina riduca il rischio di eventi ischemici ricorrenti e che necessitano di ospedalizzazione.

Sulla base di tali considerazioni il nostro studio si propone di valutare l'impatto economico in Italia dell'utilizzo di dosi elevate di atorvastatina versus placebo in pazienti con coronaropatia.

\section{METODI}

Le informazioni cliniche sono state tratte dallo studio "Effects of Atorvastatin on Early
Recurrent Ischemic Events in Acute Coronary Syndromes" (MIRACL) [1], che ha coinvolto 3.086 pazienti di età superiore ai 18 anni, con angina instabile o con infarto acuto del miocardio, randomizzati a ricevere atorvastatina $80 \mathrm{mg} / \mathrm{die}$ (1.538) o placebo (1.548) per 16 settimane.

L'end-point primario dello studio è stato il tempo all'insorgenza di uno dei seguenti eventi: morte, ospedalizzazione per infarto miocardico acuto, arresto cardiaco non fatale o ricorrente ischemia cardiaca [1]. L'end-point secondario riguardava invece il tempo all'insorgenza di uno dei seguenti eventi: ictus non fatale, insufficienza cardiaca congestizia, angina instabile o necessità di ricorrere a una procedura di rivascolarizzazione.

La valutazione economica è stata condotta utilizzando l'analisi di costo/efficacia [2-3], confrontando le conseguenze economiche e cliniche dell'utilizzo di dosi elevate di atorvastatina $(80 \mathrm{mg} / \mathrm{die})$ versus placebo in pazienti con angina instabile o infarto acuto del miocardio.
${ }^{(1)}$ CIRFF, Centro Interdipartimentale di Ricerca in

Farmacoeconomia e Farmacoutilizzazione, Università degli Studi di Napoli Federico II 


\section{Tabella I \\ Costi unitari \\ * Media ponderata per la frequenza di ospeda- lizzazioni come risulta dalle schede di dimis- sione ospedaliera (SDO 2003) [8]}

\begin{tabular}{lcc}
\hline \multicolumn{1}{c}{ Variabili } & DRG [7] & Costi unitari (euro) \\
\hline Infarto miocardico non fatale & 121,122 & $4.249,49^{*}$ \\
Infarto miocardico fatale & 123 & $3.510,87$ \\
Arresto cardiaco con rianimazione & 129 & $4.040,76$ \\
Ictus fatale e non fatale & 14 & $3.926,62$ \\
Rivascolarizzazioni coronariche & $106,107,112$ & $7.996,28^{\star}$ \\
Angina instabile & 140 & $2.179,45$ \\
Insufficienza cardiaca congestizia non fatale & 127 & $3.091,51$ \\
Atorvastatina (80 mg) & - & 1,56 \\
\hline
\end{tabular}

L'analisi è stata eseguita secondo la prospettiva del Servizio Sanitario Nazionale (SSN) italiano, adottando il profilo temporale dello studio MIRACL che indica 16 settimane di follow-up.

Tutte le analisi sono riferite ad ipotetiche coorti di 1.000 pazienti [4-5].

È stata pertanto condotta un'analisi di costo/ efficacia, calcolando il rapporto incrementale di costo/efficacia (ICER) come rapporto tra la differenza nei costi delle due alternative in relazione alla differenza di efficacia. L'ICER è espresso come costo per evento evitato.

\section{Effetti}

Sono stati identificati, misurati e quantificati gli effetti mediante l'utilizzo delle evidenze dello studio MIRACL, espresse con le seguenti misure: eventi evitati.

\section{Costi}

Coerentemente con la prospettiva d'analisi, sono stati identificati, misurati e quantificati i costi diretti sanitari.

Per il calcolo del costo della terapia farmacologica si è utilizzato lo schema terapeutico seguito nello studio MIRACL. Tale schema prevedeva che i pazienti che rispettavano i criteri di eleggibilità venivano randomizzati a ricevere $80 \mathrm{mg} /$ die di atorvastatina o placebo per 16 settimane.

Alla fine del periodo di follow-up lo $0,5 \%$ nel gruppo con atorvastatina e lo $0,2 \%$ nel gruppo con placebo sono usciti dallo studio [1].

Il costo della terapia farmacologia è stato quantificato sulle basi della dose giornaliera e della durata del trattamento, utilizzando il prezzo di cessione all'SSN dei farmaci e supponendo che il prezzo di atorvastatina $80 \mathrm{mg}$ sia uguale a quello di atorvastatina $40 \mathrm{mg}$ [6].

I costi delle ospedalizzazioni sono stati calcolati sulla base delle tariffe ospedaliere associate ai DRG (Diagnosis Related Group) [7], scelta consistente con la prospettiva adottata. Le tariffe associate ad ogni evento clinico sono riportate in Tabella I. In relazione alla gravità dello stato di salute del paziente alla dimissione potrebbero esistere più tariffe associate a quel tipo di evento clinico, come per esempio nel caso della rivascolarizzazione; in tal caso, poiché dati sulla gravità non erano disponibili, si è utilizzato il costo medio pesato per la frequenza di evento in accordo con le più recenti informazioni disponibili sui ricoveri ospedalieri in Italia [8].

I costi associati agli eventi avversi da farmaco non sono stati considerati nell'analisi in quanto non erano disponibili informazioni dettagliate a riguardo.

I costi sono espressi in euro 2006.

\section{Sensibilità}

Al fine di determinare la solidità dell' analisi è stata condotta un'analisi di sensibilità sui principali parametri: prezzo dei farmaci $( \pm 10 \%)$, costi delle ospedalizzazioni e delle procedure $( \pm 10 \%)$ [9-11].

\section{RISULTATI}

Lo studio MIRACL voleva dimostrare come una strategia atta a ridurre il colesterolo produce benefici. I risultati mostrano come il trattamento con atorvastatina $80 \mathrm{mg}$ riduce il rischio di ricorrenza di eventi ischemici.

Lo studio però non presentava la potenza necessaria per rilevare le differenze tra i due gruppi per i singoli componenti dell'end-point primario composto. Anche se le morti, l'infarto miocardico acuto e l'arresto cardiaco ricorrevano meno frequentemente nel gruppo in trattamento con atorvastatina, le differenze non erano statisticamente significative. La maggior parte delle differenze tra gruppi nell'end-point primario combinato riguardano la riduzione delle ischemie del miocardio ricorrenti che necessitavano di una ospedalizzazione immediata. Il numero di ictus era inferiore nel gruppo trattato con atorvastatina, suggerendo che tale farmaco produce un beneficio ulteriore sugli eventi cerebrovascolari, anche se il numero degli ictus era basso in ogni gruppo.

Il costo della terapia con atorvastatina in 16 settimane è stato di circa 162.489.000 euro per 1.000 pazienti. I costi totali delle ospedalizzazioni per eventi fatali e non è stato stimato pari a 2,1 milioni di euro nel gruppo trattato con 


\begin{tabular}{lccccc}
\hline \multirow{2}{*}{ Variabili } & \multicolumn{2}{c}{ Placebo* } & \multicolumn{2}{c}{ Atorvastatina* } & \multirow{2}{*}{ Differenza* } \\
\cline { 2 - 5 } & Numero & Costi totali & Numero & Costi totali \\
\hline Infarto miocardico non fatale & 73 & $310.212,94$ & 66 & $280.466,50$ & $-29.746,45$ \\
Infarto miocardico fatale & 44 & $154.478,28$ & 42 & $147.456,54$ & $-7.021,74$ \\
Arresto cardiaco con rianimazione & 10 & $40.407,60$ & 8 & $32.326,08$ & $-8.081,52$ \\
Ictus fatale e non fatale & 16 & $62.825,92$ & 8 & $31.412,96$ & $-31.412,96$ \\
Rivascolarizzazioni coronariche & 161 & $1.287 .401,08$ & 165 & $1.319 .386,20$ & $31.985,12$ \\
Angina instabile & 68 & $148.202,60$ & 59 & $128.587,55$ & $-19.615,05$ \\
Insufficienza cardiaca congestizia non fatale & 28 & $86.562,28$ & 26 & $80.379,26$ & $-6.183,02$ \\
Costi totali per ospedalizzazioni & & $\mathbf{2 . 0 9 0 . 0 9 0 , 7 0}$ & & $\mathbf{2 . 0 2 0 . 0 1 5 , 0 9}$ & $\mathbf{- 7 0 . 0 7 5 , 6 2}$ \\
Costo terapia farmacologica & & $\mathbf{0}$ & & $\mathbf{1 6 2 . 4 8 9 , 6 0}$ & $\mathbf{1 6 2 . 4 8 9 , 6 0}$ \\
Costi totali & & $\mathbf{2 . 0 9 0 . 0 9 0 , 7 0}$ & & $\mathbf{2 . 1 8 2 . 5 0 4 , 6 9}$ & $\mathbf{9 2 . 4 1 3 , 9 8}$ \\
Qualsiasi evento & $\mathbf{3 0 7}$ & & $\mathbf{2 9 3}$ & & $\mathbf{- 1 4}$ \\
ICER & & & & & $\mathbf{6 . 6 0 1}$ \\
\hline
\end{tabular}

Tabella II

Analisi di costo/efficacia

* Dati riferiti ad una ipotetica coorte di 1.000 soggetti

placebo e pari a 2,02 milioni di euro nel gruppo trattato con atorvastatina (Tabella II).

Nel complesso il costo per 1.000 pazienti trattati per un periodo di 16 settimane è stato stimato in 2.090.090,7 euro nel gruppo placebo e di 2.182.504,69 euro nel gruppo trattato con atorvastatina $80 \mathrm{mg}$.

Il costo totale dovuto alla terapia con atorvastatina $80 \mathrm{mg}$ in soggetti affetti da coronaropatia è di circa 2,2 milioni di euro per 1.000 pazienti trattati, con un rapporto incrementale di costo/efficacia (ICER) di 6.601 euro per evento evitato (Tabella II).

Si è osservata una variazione del rapporto incrementale al variare del costo del farmaco e delle ospedalizzazioni (Tabella III). L'ICER passa infatti da 5.440,36 euro a 7.761,64 euro per evento evitato se cambia il costo giornaliero di atorvastatina, mentre passa da 6.100,46 euro a 7.101,54 euro quando si applica una variazione ai costi delle ospedalizzazioni.

\section{DISCUSSIONE}

Il presente studio è la prima valutazione economica dello studio MIRACL adattato alla realtà italiana, non sono infatti disponibili studi simili di confronto a livello italiano.

Si è dimostrato come il trattamento con elevate dosi di atorvastatina porta ad una riduzione dei costi diretti a carico dell'SSN, anche se non tale da compensare interamente il costo addizionale dovuto al trattamento con atorvastatina $80 \mathrm{mg}$.

Lo studio presenta potenziali limitazioni: ad esempio sono calcolati solo i costi delle ospedalizzazioni e non i costi associati alla riabilitazione e alla gestione del paziente fuori dalla struttura ospedaliera. Tali informazioni non sono registrate.

Una seconda limitazione è rappresentata dal fatto che le conseguenze indirette di un evento cardiovascolare quali la perdita o il guadagno di produttività o conseguenze intangibili quali la compromissione della qualità di vita associata allo stato di salute non sono state incluse. Il motivo è legato al fatto che queste informazioni non erano a nostra disposizione. Sarebbe comunque interessante valutare come la terapia con statine possa influenzare la produttività dei pazienti. Ad ogni modo è possibile ipotizzare che i pazienti in trattamento con atorvastatina $80 \mathrm{mg}$ abbiano subìto una minore riduzione nella capacità lavorativa, a causa delle meno frequenti ospedalizzazioni. Per questo motivo l'introduzione nella valutazione economica anche dei costi indiretti aumenterebbe i benefici economici derivanti dall'utilizzo di dosi elevate di atorvastatina.

In conclusione riteniamo opportuno sottolineare che il costo della terapia farmacologica è stato probabilmente sovrastimato: infatti, per mancanza di informazioni relative alla durata del ricovero, non è stato possibile tenere in considerazione che il costo della terapia farmacologica è compreso nelle tariffe DRG per il periodo della durata del ricovero. Per tale motivazione una parte dei costi relativi alla terapia sono stati quantificati due volte.

\begin{tabular}{lcc}
\hline \multicolumn{1}{c}{ Descrizione } & Risultati (euro) & Variazione \% \\
\hline Prezzo atorvastatina $\pm 10 \%$ & $5.440,36-7.761,64$ & $\pm 17,58$ \\
Costo ospedalizzazioni $\pm 10 \%$ & $6.100,46-7.101,54$ & $\pm 7,58$ \\
\hline
\end{tabular}

\section{Tabella III}

Analisi di sensibilità 


\section{BIBLIOGRAFIA}

1. Schwartz GG, Olsson AG, Ezekowitz MD, Ganz P, Oliver MF, Waters D et al. Effects of atorvastatin on early recurrent ischemic events in acute coronary syndrome: the MIRACL study: a randomized controlled trial. JAMA 2001; 285: 1711-8

2. Weinstein Mc, Stason WB. On the foundations of cost-effectiveness analysis for health and medical practices. $N$ Engl J Med 1977; 296: 716-21

3. Drummond MF, Stoddart GL, Torrance GW. Methods for economic evaluation of health care programmes. Oxford: Oxford University Press, 1997

4. Mantovani LG, Belisari A, Dobrilla G. Valutazione economica del lansoprazolo nel trattamento dei pazienti affetti da malattia da reflusso esofageo. PharmacoEconomics Italian Research Articles 1999; 1: 43-51

5. Scalone L, Mantovani LG. Valutazione economica della terapia con lisinopril ad alto verso basso dosaggio nel trattamento dei soggetti con scompenso cardiaco cronico. Pharmacoeconomics Italian Research Articles 2002; 4; 45-55

6. L'informatore farmaceutico. Milano: OEMF, 2006

7. Decreto ministeriale 14/12/94 in tema di "Tariffe delle prestazioni di assistenza ospedaliera". Supplemento ordinario alla Gazzetta ùUfficiale n. 209 del 8/09/1997

8. http://www.ministerosalute.it/programmazione/sdo/sdo.jsp

9. Briggs A, Sculpher M, Buxton M. Uncertainty in the economic evaluation of health care technologies: the role of sensitivity analysis. Health Econ 1994; 3: 95-104

10. Garattini L, Grilli R, Scopelliti D, Mantovani L. A proposal for Italian guidelines in pharmacoeconomics. Pharmacoeconomics 1995; 7: 1-6

11. Capri S, Ceci A, Terranova L, Merlo F, Mantovani LG. Guidelines for economic evaluation in Italy: recommendation from the Italian group of pharmacoeconomic studies. Drug Inf J 2001; 35: 189-201 